3D analysis of synaptic vesicle density and distribution after acute foot-shock stress by using serial section transmission electron microscopy

Khanmohammadi, Mahdieh; Darkner, Sune; Nava, Nicoletta; Nyengaard, J. R.; Wegener, G.; Popoli, M.; Sporring, Jon

Published in:

Journal of Microscopy

DOI:

10.1111/jmi.12468

Publication date:

2017

Document version

Early version, also known as pre-print

Citation for published version (APA):

Khanmohammadi, M., Darkner, S., Nava, N., Nyengaard, J. R., Wegener, G., Popoli, M., \& Sporring, J. (2017).

$3 \mathrm{D}$ analysis of synaptic vesicle density and distribution after acute foot-shock stress by using serial section transmission electron microscopy. Journal of Microscopy, 265(1), 101-110. https://doi.org/10.1111/jmi.12468 


\title{
3D analysis of synaptic vesicle density and distribution after acute foot-shock stress by using serial section transmission electron microscopy
}

\author{
Mahdieh Khanmohammadi ${ }^{1, *}$, Sune Darkner, Nicoletta Nava, Jens Randel \\ Nyengaard \\ Translational Neuropsychiatry Unit, Department of Clinical Medicine, Aarhus \\ University, Denmark, Denmark \\ Gregers Wegener \\ Translational Neuropsychiatry Unit, Department of Clinical Medicine, Aarhus \\ University, Denmark, Denmark; Pharmaceutical Research Center of Excellence, School \\ of Pharmacy, North-West University, Potchefstroom, South Africa \\ Maurizio Popoli \\ Laboratory of Neuropsychopharmacology and Functional Neurogenomics - Dipartimento \\ di Scienze Farmacologiche e Biomolecolari and Center of Excellence on \\ Neurodegenerative Diseases, Universita degli Studi di Milano, Milano, Italy \\ Jon Sporring \\ Computer Science Department, University of Copenhagen, Denmark
}

\begin{abstract}
Behavioral stress has shown to strongly affect neurotransmission within the neocortex. In this study, we analysed the effect of an acute stress model

\footnotetext{
${ }^{*}$ Corresponding Author

Email addresses: mahdieh@di.ku.dk (Mahdieh Khanmohammadi), darkner@di.ku.dk (Sune Darkner), nicoletta.nava@clin.au.dk (Nicoletta Nava), jrnyengaard@clin.au.dk (Jens Randel Nyengaard), wegener@clin.au.dk (Gregers Wegener), maurizio.popoli@unimi.it (Maurizio Popoli), sporring@di.ku.dk (Jon Sporring)

${ }^{1}$ Computer Science Department, Sigurdsgade 41, 2100 Copenhagen, Denmark; Tel.: +45-50123164; Fax: +45-3432-1401
} 
on density and distribution of neurotransmitter-containing vesicles within medial prefrontal cortex. Serial section transmission electron microscopy was employed to compare two groups of male rats: 1) rats subjected to foot-shock stress and 2) rats with sham stress as control group.

2 dimensional (2D) density measures are common in microscopic images and are estimated by following a two-dimensional path in-section. However, this method ignores the slant of the active zone and thickness of the section. In fact, the active zone is a surface in three-dimensions (3D) and the 2D measures do not accurately reflect the geometric configuration unless the active zone is perpendicular to the sectioning angle.

We investigated synaptic vesicle density as a function of distance from the active zone in 3D. We reconstructed a 3D dataset by estimating the thickness of all sections and by registering all the image sections into a common coordinate system. Finally, we estimated the density as the average number of vesicles per area and volume and modelled the synaptic vesicle distribution by fitting a one-dimensional parametrised distribution that took into account the location uncertainty due to section thickness.

Our results showed a clear structural difference in synaptic vesicle density and distribution between stressed and control group with improved separation by $3 \mathrm{D}$ measures in comparison to the $2 \mathrm{D}$ measures. Our results showed that acute foot-shock stress exposure significantly affected both the spatial distribution and density of the synaptic vesicles within the pre-synaptic terminal.

Keywords: Synaptic vesicle density; distribution modeling; acute foot-shock stress; serial section transmission electron microscopy; specimen thickness estimation; image registration.

\section{Introduction}

Synapses are the sites where information flows from one neuron to another. Upon arrival of an action potential at the nerve terminal, voltagegated $\mathrm{Ca}^{2+}$ channels open; the rapid rise in intracellular $\mathrm{Ca}^{2+}$ triggers exocytosis of synaptic vesicles and the release of neurotransmitter at a specialised site of the presynaptic compartment termed active zone. A number of studies have recently shown that behavioral stress may strongly impact the release of neurotransmitter-containing vesicles from presynaptic terminals of medial prefrontal cortex (mPFC) (McEwen and Gianaros, 2010). In particu- 
lar, acute stressors of diverse type, have shown to rapidly enhance glutamate transmission within the prefrontal cortex (Moghaddam, 1993; Treccani et al, 2014; Musazzi et al, 2010; Satoh and Shimeki, 2010).

Given the primary role of synaptic vesicles as mediators of neurotransmitter release, we hypothesise that the enhancement of glutamate release induced by acute stressors correlates directly with statistical distribution of glutamate-containing vesicles and their distances from the active zone. To verify our hypothesis, we first subjected rodents to acute foot-shock (FS)stress, a behavioral model shown to produce increase glutamate release within mPFC (Vollmayr and Henn, 2001).

We measure the density of the synaptic vesicle as a function of distance from the active zone in order to model their statistical distribution in the pre-synaptic terminal. A common estimate of the density of vesicles as a function of distances from the active zone is the in-section estimated density. However, the active zone is a $3 \mathrm{D}$ surface and the in-section, $2 \mathrm{D}$, distance is only a good approximation of the shortest distance, when the active zone is near perpendicular to the cutting direction. Hence, we propose to reconstruct the synapse in 3D and estimate the true shortest distance.

ssTEM projects the content of section of uncertain thickness into images; this, in turn, can influence the estimated parameters in 3D. We use a newly presented method for estimating the section thickness using image statistics only (Sporring et al, 2014), and we present a novel method for estimating the densities of vesicles taking into account the projection uncertainty. We previously showed that the acute FS-stress significantly increases the number of docked vesicles in mPFC as compared to sham-stress (Nava et al, 2015). Here, we hypothesise that the synaptic vesicles distribution can be modeled by a one-dimensional exponential distribution, parameterised by the shortest distance to the active zone.

This paper contributes with the following: 1) Proposing an algorithm for estimating 3D distances and densities of synaptic vesicles. 2) Comparing the 2D and 3D measurements on synaptic vesicle density and distribution; 3) Showing that the density of synaptic vesicles decays faster by distance from the active zone in FS-stressed rats as compared to sham rats and that synaptic vesicles are more compact around the active zone in FS-stressed rats as compared to control animals; 4) Showing a clear difference between the two experimental groups specially in $3 \mathrm{D}$ estimation as compared to $2 \mathrm{D}$ estimations. 


\section{Materials}

The datasets were obtained from male Sprague-Dawley rats (300-315g), using a FEI Morgagni TEM and SIS Megaview III digital camera at Aarhus University (Nava et al, 2014). Rats were housed two per cage under a $12 \mathrm{~h}$ light/dark cycle, at room temperature $\left(22^{\circ} \mathrm{C}\right)$ and humidity, with food and water ad libitum. The animals were randomly sorted into two experimental groups (6 per group): rats subjected to sham stress and rats subjected to acute FS-stress. Soon after stress induction, anaesthetised animals were perfused transcardially with heparinised saline followed by $2.5 \%$ glutaraldehyde and $2 \%$ paraformaldehyde in $0.1 \mathrm{M}$ cacodylate buffer $\left(\mathrm{pH}=7.4,4^{\circ} \mathrm{C}\right)$. Brains were removed from the skull, weighed and postfixed in the same fixative at $4^{\circ} \mathrm{C}$. Left or right hemisphere was randomly selected and embedded in $5 \%$ agar. From the rostral surface, $100 \mu \mathrm{m}$ thick sections were cut coronally on a Vibratome 3000 (Vibratome, St Louis, MO). Two sets of 13/14 sections each were randomly selected based on a systematic random sampling principle and a sampling fraction of $1 / 6$. mPFC was identified based on its noticeable cytoarchitectural features (Van Eden and Uylings, 1985). Specimens were encapsulated in ultra-hard epon blocks and were manually cut into series of $45 \pm 15 \mathrm{~nm}$ thick sections, where the variation was caused by the manual cutting process. Each section was collected on a pioloform-coated slot formvar film, and the formvar film was visualised in the microscope (Nava et al, 2014). The image pixel size was $2.5 \times 2.5 \mathrm{~nm}^{2}$. For each series of sections, synapses free from artefacts were manually selected, tracked and imaged across sections. The sequences of images with complete glutamatergic boutons were identified and relevant structures were delineated. Two experimenters blind to animal conditions performed the manual selection and annotations of sections (NN, MKh). Two annotated excitatory synapses identified from the dataset are shown in Figure 1.

[Figure 1 about here.]

Finally, to study the intensity inhomogeneity, 10 blank images (no specimen in the microscope) were taken in rapid succession without changing the microscope parameters.

\section{Methods}

We propose a multi-stage method: starting with intensity inhomogeneity correction; followed by affine image registration; estimation of section thick- 
ness; manual annotation of vesicles, active zones and pre- and post-synaptic terminals; fitting the active zones with a cubic spline surface; calculating the densities using two different methods; and finally modeling the distribution by a one-dimensional parametrised model.

\subsection{Preprocessing of the Data}

The imaging method introduces severe noise and artefacts, which must be reliably suppressed or avoided in order to gain consistent information. The sources of noise we have observed were 1) The cutting process, 2) the formvar film, and 3) the electron microscope camera system employed for section visualisation, Figure 2. Synaptic regions containing artefacts from cutting process and/or from formvar film were discarded.

[Figure 2 about here.]

Figure 2 shows an inhomogeneity in image intensity, also known as bias field; this does not seem to relate to the content of the section. To investigate the nature of this inhomogeneity we acquire blank images (no material in the microscope): an example is shown in Figure 2(c). These "empty" images were fitted a quadratic polynomial and the original image was subtracted the fit to produce the bias-normalised image. To verify whether the estimated bias field is additive or multiplicative, we subdivided the image domain into 9 equal size and non-overlapping regions and estimated the histograms of the 4 corners and the center. These local histograms were compared to each other as well as the global histogram using Student's T-test. Further, in a sample image from real data with specimen, we defined several random intensity bands and checked the intensity variance in those bands. The intensity bands were defined in such a way that the sample number in each band was approximately the same. We estimated the variance for around 50 randomly picked intensity bands such as, $40<I<50,100<I<110,150<I<160$, $215<I<225$, etc..

To realign the serial sections for 3 dimensional analysis of the original samples we first established their relation in the $x$ - and $y$-direction using image registration. We aligned the sections pairwise using affine deformations of the underlying space using a standard registration technique (Darkner and Sporring, 2013) and subsequently mapped the images into a common coordinate system by composing their pair-wise relations. The registration method is an energy minimisation of a dissimilarity. We used sum of the 
pixelwise difference of the absolute difference between the two images. We further augmented the algorithm in (Darkner and Sporring, 2013) with a continuation method, where we initially registered the images smoothed with a large Gaussian, $G(x, \sigma)=\frac{1}{2 \pi \sigma^{2}} e^{\frac{-x^{2}}{2 \sigma^{2}}}$, with $\sigma=100 \mathrm{~nm}$. Using the estimated transformation as the initial point, we then registered the same images but smoothed with a smaller Gaussian with $\sigma=50 \mathrm{~nm}$, and this was repeated with $\sigma=25,10$, and $5 \mathrm{~nm}$.

To establish the relation between the sections in the $z$-direction, we estimated the thickness of each section using (Sporring et al, 2014): the distance between two registered images was estimated by first normalising the average intensity value to be 0 and the standard deviation to be 1 for each image. Then the standard deviation of the difference between pixel values as a function of distance between the pixels for each image was calculated, and these two scaling functions were averaged. Finally, the standard deviation of the difference between the pixel in one image and the other directly above was calculated and using an inverse lookup in the average scaling function we estimated the distance between images in $x$-pixel units.

Finally, a randomly selected set of excitatory glutamatergic synapses was identified, where complete active zones could be clearly identified and were free from other artefacts. For these synapses, active zone, pre-synaptic terminal boundary and center of vesicles were manually annotated as sequences of points in each section.

\subsection{Non-Parametric Density Estimation in 2 and 3 Dimensions}

For 2D analysis, the sections were treated independently; the points corresponding to the active zone and the boundary of the pre-synaptic terminal in each section were then fitted with 2D cubic spline curves. Parallel curves of the active zone were generated at interval of $50 \mathrm{~nm}$ until $200 \mathrm{~nm}$ using a variation of the signed distance algorithm (Osher and Fedkiw, 2003). Our version calculated for each pixel location the shortest distance to the active zone. Intervals of $50 \mathrm{~nm}$ were chosen to be similar to the average vesicle size, and the maximum of $200 \mathrm{~nm}$ was chosen so that the influence of mitochondria on the histograms could be avoided in our images. The parallel curves were confined to the pre-synaptic terminal boundary to produce closed regions as illustrated in Figure 3.

[Figure 3 about here.] 
Then we calculated the 2D density of synaptic vesicles as the number of vesicle centers inside each region divided by the area of the region. Finally, we calculated the per region average density for each synapse.

In 3D, each synapse was treated independently, i.e., for a single synapse, the set of annotations across related sections for the active zone and the pre-synaptic terminal were fitted with cubic splines. 3D distance fields were generated in a similar manner as in 2D but using 3D distances, and parallel equidistance surfaces $50 \mathrm{~nm}$ from the active zone and limited by the presynaptic terminal. The 3D density was estimated as the number of vesicle centers inside the closed 3D volumes divided by the volume.

Finally, in order to estimate the approximate error in the 2D density estimates, we approximated the 3D active zones with planes and calculated the angle between the fitted plane's normal and the $z$-direction.

\subsection{Parametrised Model of Synaptic Vesicle Distribution}

A one-parameter model was developed as an alternative measure for the distribution of vesicles near the active zone, which explicitly models the effect of thickness and object projection in ssTEM. A simplified example of the relation between the active zone (a plane), a specimen section, vesicles, and the parallel planes w.r.t the active zone is illustrated in Figure 4(a).

[Figure 4 about here.]

A side view concentrating on a single vesicle is shown in Figure 4(b) and illustrates the geometry of projection and distance estimation: consider a set of vesicles, whose centers are denoted as $\boldsymbol{x} \in \mathbb{R}^{3}$, an active zone modeled as a plane and with a unit normal $\boldsymbol{n}$ and containing a reference point $\boldsymbol{x}_{0}$, and thick section with the top and bottom of the section given as 2 parallel planes $h$ apart and with identical unit normals $\boldsymbol{m}$ that is angled $\theta=\pi / 2-\phi$ with respect to $\boldsymbol{n}$, where $\phi$ is the angle between the section and the active zone normal. The centroid of the synaptic vesicles in the images represent a set of projections $\boldsymbol{z}_{i}$ of vesicles $\boldsymbol{x}_{i}$ onto the lower plane of a section, the image plane, along $\boldsymbol{m}$. We define an observation window $a<d<b$ for the observed shortest distances from all $\boldsymbol{z}_{i}$ to the active zone, where $a$ is the minimum of the observations and $b$ is the maximum of the observations.

We used the method of moments to estimate the parameters of $p$, the synaptic vesicles distribution: let $\alpha(\boldsymbol{x})=\boldsymbol{n} \cdot\left(\boldsymbol{x}-\boldsymbol{x}_{0}\right)$ be a function for estimating the shortest distance from a vesicle centroid to the active zone, 
and assume that $p$ belongs to a one parameter family of distributions in direction $\boldsymbol{n}$ and uniform orthogonal to $\boldsymbol{n}$, then the average observed distances is $\bar{\alpha}=\frac{1}{N} \sum_{i=1}^{N} \alpha_{i}$, where $\alpha_{i}=\alpha\left(\boldsymbol{z}_{i}\right), a<\alpha_{i}<b$ is the observed distance of vesicle $i$, whose true location is $\boldsymbol{x}_{i}$. Hypothesising an integrable density function $p$, the projections along $\boldsymbol{m}$ induces a distribution on the bottom of a section as,

$$
f(\alpha)=\frac{1}{\sin \phi} \int_{\alpha}^{\alpha+h \sin \phi} p(\beta) d \beta
$$

If the section is perpendicular to the active zone, $\phi=0$, then the real distribution of vesicles $p$ equals the distribution of observations $f$ due to projections. The expected value of the average observed projected distances is,

$$
\mu=\frac{\int_{a}^{b} \alpha f(\alpha) d \alpha}{\int_{a}^{b} f(\alpha) d \alpha}
$$

We solved for the parameter of $p$ such that $\bar{\alpha}=\mu$ with respect to the parameter of the distribution.

Previous studies have shown that the exponential distribution is a good fit to the synaptic vesicles distribution (Khanmohammadi et al, 2014), thus we chose $p(x)=\lambda e^{-\lambda x}$, in which case,

$$
\mu=\frac{e^{a \lambda}(b \lambda+1)-(a \lambda+1) e^{b \lambda}}{\lambda\left(e^{a \lambda}-e^{b \lambda}\right)}
$$

Interestingly enough, this equation is independent of $\phi$, but of course $\phi$ influences the observed distances. For this distribution, $\bar{\alpha}=\mu$ is a nonlinear equation, so to solve for $\lambda$ we formulated a minimisation problem as, $\hat{\lambda}=\arg \min (\bar{\alpha}-\mu)^{2}$ with respect to the parameter of the distribution. We employed this method to estimate the parameter $\lambda$ of the distribution for $2 \mathrm{D}$ and $3 \mathrm{D}$ measurements of the shortest distance from synaptic vesicles to the active zone.

The accuracy of the obtained equations of parametrised model for distribution were evaluated by conducting a computer simulation. We randomly drew 10000 points in a region of $[0, \infty) \times[0,300] \times[0,300]$, which were distributed as an exponential distribution in $\alpha$ with $\lambda=0.01$ and uniformly in the two other directions. Of this set we cut a single section of height $h=50$ at various angles in such a way that boundary artefacts were reduced. For each angle, we projected the samples down to the bottom of the section, and 
determined $a$ as the projection of the length of the projection of the active zone in the section, and set $b=100$. Projections with distances outside the interval $[a, b]$ were discarded.

\section{Results}

The proposed method was applied to 11 sets of ssTEM images obtained from $\mathrm{mPFC}$ of rodents subjected to sham-stress $(n=6)$ and to acute FSstress $(n=5)$. Due to presence of corrupted images, dataset from one rodent subjected to acute FS-stress were excluded. Overall, at least one excitatory synapse was included for each rat in the study. Only excitatory boutons with active zone and presynaptic terminal entirely included in the series of sections were chosen for this study: a total of 15 synapses were included. Small synapses include 4 sections and larger ones could include up to 10 sections. In total 51 sections were employed for extraction of synapses from sham rats, and 37 sections from stressed rats.

\subsection{Quality Inspection of the Preprocessing}

For the empty images, the local histograms of the 5 non-overlapping regions and their global histogram showed p-value less than $10^{-10}$ using Student's T-test. For the specimen images, the analysis of the variance of the intensity values in the intensity bands showed only little variation with position in the image.

An example on the effect of registration is shown in Figure 5 using the chessboard visualisation technique, where the black pieces of the chessboard shows the first image and the white pieces show the second image. As illustrated in the figure, the boundaries of the objects are better aligned after registration in Figure 5(b).

[Figure 5 about here.]

\subsection{Non-Parametric Density Estimation in 2 and 3 Dimensions}

Figure 6 shows the mean 2D and 3D estimated density in FS-stressed rats compared with sham rats. The $2 \mathrm{D}$ densities were estimated in a distance map in every section of each synapse and were then averaged over all synapses per group. The 3D densities were estimated on the same sections as used for the $2 \mathrm{D}$ densities, but after $3 \mathrm{D}$ reconstruction and 3D distance computations. These were also averaged per group. 
[Figure 6 about here.]

We observed that both 2D and 3D density estimates showed higher density of vesicles near the active zone. Both clearly separate the two groups of rats, however, the $3 \mathrm{D}$ density estimates showed improved separation over $2 \mathrm{D}$ especially near the active zone.

The average slope of the active zone surface was estimated by fitting a plane to it. Table 1 reports estimated average angles in the synapses included in this study.

[Table 1 about here.]

The average angles show small variation from the right angle.

\subsection{Parametrised Model of Synaptic Vesicle Distribution}

The result on the quality of the parametrised model was assessed through simulations of the mentioned synthetic experiment in section 3.3. Two estimates of $\lambda$ were computed: $\lambda_{2 \mathrm{D}}$ and $\lambda_{3 \mathrm{D}}$. For $\lambda_{2 \mathrm{D}}$ we only considered the projections in a single section, where the positions of the active zone is set to be the middle of the projection of the part of the active zone intersecting with the section, and the distances were calculated in section ignoring 3D information. For $\lambda_{3 \mathrm{D}}$ we fitted a plane to the middle of projections of consecutive active zones. Then we calculated the shortest distance in $3 \mathrm{D}$ from the projected vesicles to the fitted plane and corrected with $(-1)^{-\operatorname{sgn}(\phi)} h \sin (\phi) / 2$ to account for the geometric bias. Statistics for 100 experiments are given in Table 2 .

[Table 2 about here.]

From the table we observed that the two methods of estimating $\lambda$ give similar results, when the angle is close to 0 , but deviate considerably for large angles. We also observed that the 3D method gives result close to the generating $\lambda$ except for extreme angles.

In real data, we fitted a parametric model to the distribution of the synaptic vesicles in each synapse. Figure 7 shows the results of the estimated lambda of the fitted model to the 2D and 3D distances for each synapse. The median of the estimated parameter $\lambda$ for the two groups of rats are shown as solid lines in the figure. The estimated medians in 2D are $\lambda_{\text {Sham }}=0.0067$ and $\lambda_{F S-\text { stress }}=0.012$ and in $3 \mathrm{D}$ are $\lambda_{\text {Sham }}=0.0053$ and $\lambda_{F S-\text { stress }}=0.0137$. We observed that the medians indicate the difference between the two groups 
of rats very well, and that the 3D estimations provided better separation between the groups of rats compared to that of 2D estimations. We also observed that the parameter $\lambda$ were in general larger for FS-stressed rats compared to the control rats.

[Figure 7 about here.]

Finally, in 2D we identified S4, S6, S7, S8, and S10 to be outliers with respect to the fit, while the number of outliers in 3D were reduced to $\mathrm{S} 4, \mathrm{~S} 6, \mathrm{~S} 7$ and S8.

\section{Discussion}

At the synaptic level, neurotransmitter-containing vesicles occupy a $3 \mathrm{D}$ space, but typical imaging methods can only visualize their location with respect to the active zone as $2 \mathrm{D}$ images. In this paper we have studied vesicle distribution through ssTEM microscopy as this modality is able to acquire images with very high resolution. However, ssTEM relies on physically sectioning of material from a volume by which the relation between sections is lost. Image registration is a standard method for realigning images in $x$ - and $y$ - direction and we have used this method here with success. Considering the hardness of the encasing material and visual inspections, we have concluded that the main mode of variation between sections comes from manual handling, hence an affine motion model is sufficient. However, any method relying on physical sectioning of material must also take into account the uncertainty of the cutting process. In our work, the sections were ultra thin $\sim 45 \mathrm{~nm}$ and no standard technique for estimating their thickness existed. We have therefore used a recently developed method that uses in-image and between images statistics to estimate the apparent distance between neighbouring images. Due to lack of ground truth data the validity of this method can only be assessed through synthetic experiments and by visual inspection of 3D surfaces (Sporring et al, 2014). Thus, the issue is whether the assumptions in the synthetic experiments are valid for the real data. Our view is that besides the before mentioned indications of precision, the relevant scaling function used appears very stable for the present image class and that the distance estimates appear to improve the separation between groups. Hence, we assume that the estimated $z$-positions of the sections are useful.

One of the main issues when statistically modelling vesicle distribution is to find parameters that describes their location regardless of the great variability of pre-synaptic region shapes and despite the fact that we only have 
snapshots of their motion. It is well established that the prime nature of the vesicles is to transport their neurotransmitter to the active zone and that the biological setup is tuned for this process to be fast. Hence, it seems reasonable to include the shortest distance from any point inside the pre-synaptic region to the closest point on the active zone. Another observation is that pre-synaptic regions vary a lot in size and this is somewhat linked to the size of the active zone. Since the number of vesicles that can transmit neurotransmitters through an active zone is limited by its size we hypothesise that the number of vesicles that are received per active zone per unit of time on average is proportional to the size of the active zone. Thus, we compare the distribution of vesicles for small and large active zones directly by calculating the average number of vesicles in parallel distance-bands divided by the area in 2D and volume in 3D near the active zone. The usefulness of these size- and shape-normalisations are best validated through the separation of groups, where previous studies have shown clear differences and our results confirm such findings (Nava et al, 2015; Treccani et al, 2014; Khanmohammadi et al, 2014). Thus, we conclude that distance and active zone area are important parameters for modelling vesicle locations around the active zone.

In this work we have considered 2 methods for estimating the vesicle density as a function of distance to the active zone: binning and fitting with an exponential distribution. The binning method in 3D appears attractive, since it includes the section thickness in estimating the shortest distance between the vesicles and the active zone, however in this method the position of vesicles along the hight of the section is not considered. The exponential model includes the position of the vesicles along the section hight and has only 1 parameter to fit. Fewer parameters is often to be preferred, since this in general reduces the risk of overfitting models, and low order models become particularly powerful, when they are well-tuned to the underlying problem being studied. In our study, we have identified 2 concerns related to our model: 1) the difference between $2 \mathrm{D}$ and $3 \mathrm{D}$ is less than expected, and 2) the exponential model appears to generate some outliers:

- It was somewhat surprising to us that the difference in estimated densities and exponential fits between 2 and 3D were not greater than observed as the vesicles motion is clearly unrelated to the manual sectioning direction. However, as shown in Table 1, the average angle of the active zones with respect to the sectioning direction does not stray far away from $90^{\circ}$. We hypothesise that the reason for this is that the 
apparent thickness of an active zone is proportional to its slant with respect to the angle. The active zone appears the thinnest, when it is perpendicular to the cutting direction of the specimen. This effect is shown in Figure 8.

[Figure 8 about here.]

Note also that the wide active zone shadows over the cell membrane. Hence, the technician responsible for selecting synaptic vesicles in sections may be biased towards choosing synaptic regions, where the active zone is perpendicular to the section, since these appear sharpest and the cell membrane is easily visible. Thus, the $2 \mathrm{D}$ estimated density may be good estimate and the quality may be assured by defining thickness measure that identifies the active zones that are perpendicular to the section. Nevertheless, the 3D reconstructions both avoid this complication and allow for the analysis of synaptic regions that are slanted differently with respect to the section direction.

- The number of presynaptic regions that were sampled and analysed was limited by the time-consuming preparation of specimens, and by the delicate nature of sectioning. The latter, in turn, resulted into many synapses not being entirely recovered. Also, particularly with respect to the exponential model our results showed a number of outliers. One of the outliers is synapse S7 and a sample section of this synapse is shown in Figure 9 together with a non-outlier S2.

[Figure 9 about here.]

We see that in $\mathrm{S} 7$ the active zone is very curved in contrast to $\mathrm{S} 2$, which is more flat. We hypothesise that in case of large curvature in the distance field then the induced distribution $f(\alpha)$ in (1) must be improved to integrate along curved coordinate lines instead of straight and normalised for the spreading of the implicit coordinate field generated by the curvature of the distance field.

Another outlier is the FS-stressed rat S6 shown in Figure 10.

[Figure 10 about here.] 
In our dataset the pre-synaptic region sampled from this animal appears among the most enriched in synaptic vesicles. We hypothesise that our model is challenged in this case since vesicles cannot physically overlap, while our model is not restricted to this. An improved model would take the neighbouring vesicle's pairwise interaction into account and this might better model S6.

Despite the issues discussed above, by use of robust statistics we show that the group difference is improved with the model when going from $2 \mathrm{D}$ to 3D. The developed techniques could be interesting to analyse density and distribution in other applications involving $2 \mathrm{D}$ sections.

\section{Conclusion}

In the present study, we have proposed two techniques to analyse the distribution of synaptic vesicles within $\mathrm{mPFC}$ of rats subjected to an acute stressor as compared to control animals. We hypothesise that one of the most important parameter for studying the vesicle distribution is the shortest distance to the active zone. Since this shortest distance is only approximately available in a section by section analysis, we have investigated sample synapses from both groups in the traditional section by section manner (2D) and by reconstructing synapses' 3D configuration. For both methods of analysis we have applied 2 different estimators: density binning and modelling the density by a 1 parameter exponential distribution. Our findings show that 1 ) the density clearly increases near the active zone for both groups and for all combinations of analyses and estimators confirming findings in (Khanmohammadi et al, 2014); that 2) the density around the active zone is higher in the group of rats subjected to acute FS-stress as compared to the control group for all combinations of analyses confirming findings in (Nava et al, 2015; Treccani et al, 2014); that 3) this separation between the groups is larger, when analysing the data in 3D than section by section for both estimators; and finally 4) that the estimated parameter of the exponential model of the vesicle distribution clearly separates the two groups. This work is merely methodological and the biological significance of the proposed methods needs to be addressed in a much bigger dataset, which needs further time and resources.

Future work includes developing fully automatic detection techniques, developing methods to overcome some of the gross image artefacts, developing 
automatic annotation tools, and improving the density model to better model curved distance fields and to include vesicle interactions. We also plan to investigate the effect of stress on vesicle shape and distribution in $3 \mathrm{D}$ isotropic datasets.

\section{Acknowledgement}

This research was supported by Centre for Stochastic Geometry and Advanced Bioimaging, funded by a grant from the VILLUM FOUNDATION. The authors declare no competing financial interests. We hereby confirm that none of the authors have any potential source of conflict of interest.

\section{References}

Darkner S, Sporring J (2013) Locally orderless registration. IEEE Transactions on Pattern Analysis and Machine Intelligence 35(6):1437-1450

Khanmohammadi M, Waagepetersen R, Nava N, Nyengaard J, Sporring J (2014) Analysing the distribution of synaptic vesicles using a spatial point process model. Proceedings of the 5th ACM Conference on Bioinformatics, Computational Biology, and Health Informatics pp 73-78

McEwen BS, Gianaros PJ (2010) Central role of the brain in stress and adaptation: links to socioeconomic status, health, and disease. Annals of the New York Academy of Sciences 1186(1):190-222

Moghaddam B (1993) Stress preferentially increases extraneuronal levels of excitatory amino acids in the prefrontal cortex: comparison to hippocampus and basal ganglia. Journal of neurochemistry 60(5):1650-1657

Musazzi L, Milanese M, Farisello P, Zappettini S, Tardito D, Barbiero VS, Bonifacino T, Mallei A, Baldelli P, Racagni G, et al (2010) Acute stress increases depolarization-evoked glutamate release in the rat prefrontal/frontal cortex: the dampening action of antidepressants. PloS one 5(1):e8566

Nava N, Chen F, Wegener G, Popoli M, Nyengaards J (2014) A new efficient method for synaptic vesicle quantification reveals differences between medial prefrontal cortex perforated and nonperforated synapses. Journal of Comparative Neurology 522(2):284-297 
Nava N, Treccani G, Liebenberg N, Chen F, Popoli M, Wegener G, Nyengaard JR (2015) Chronic desipramine prevents acute stress-induced reorganization of medial prefrontal cortex architecture by blocking glutamate vesicle accumulation and excitatory synapse increase. International Journal of Neuropsychopharmacology 18(3)

Osher S, Fedkiw R (eds) (2003) Level Set Methods and Dynamic Implicit Surfaces. Springer

Satoh E, Shimeki S (2010) Acute restraint stress enhances calcium mobilization and glutamate exocytosis in cerebrocortical synaptosomes from mice. Neurochemical research 35(5):693-701

Sporring J, Khanmohammadi M, Darkner S, Nava N, Nyengaard JR, Jensen EBW (2014) Estimating the thickness of ultra thin sections for electron microscopy by image statistics. Biomedical Imaging (ISBI), 2014 IEEE 11th International Symposium pp 157-160

Treccani G, Musazzi L, Perego C, Milanese M, Nava N, Bonifacino T, Lamanna J, Malgaroli A, Drago F, Racagni G, et al (2014) Stress and corticosterone increase the readily releasable pool of glutamate vesicles in synaptic terminals of prefrontal and frontal cortex. Molecular Psychiatry 19(4):433-443

Van Eden C, Uylings H (1985) Cytoarchitectonic development of the prefrontal cortex in the rat. Journal of Comparative Neurology 241(3):253267

Vollmayr B, Henn FA (2001) Learned helplessness in the rat: improvements in validity and reliability. Brain Research Protocols 8(1):1-7 


\section{List of Figures}

1 Example of section annotation. Typically, synapses present a presynaptic compartment (yellow annotation), separated from the post-synaptic terminal (cyan annotation) by an extracellular space termed synaptic cleft. Clustered within the presynaptic compartment are small translucent synaptic vesicles (blue annotation), where neurotransmitter is stored. Neurotransmitters are released upon arrival of action potential through fusion of synaptic vesicles to the active zone of the presynaptic membrane. . . . . . . . . . . . .

3 An example of producing closed parallel curves in a section. The active zone is shown in green, pre-synaptic terminal in yellow and vesicles are shown in red and blue respectively indicating location inside and outside of the closed parallel curve. 21

4 The figure shows a simplified synaptic region in 3D: a) A 3D visualisation of slicing and intersection, where the yellow thick horizontal plane illustrates a section, the active zone is illustrated as the red plane, the iso-distanced planes from the active zone are transparent red planes, and the synaptic vesicles are illustrated as blue spheres. b) A 2D visualisation of a similar situation but focussing on a single vesicle and as seen from the side. . . . . . . . . . . . . . . . . . . 22

5 Using chessboard visualisation to evaluate the registration results of two images. (a) The images before and (b) after registration. . . . . . . . . . . . . . 23

6 Histogram of synaptic vesicle density measured in (a) 2D and (b) 3D. The densities are measured in volume of interests with distance from $50 \mathrm{~nm}$ to $200 \mathrm{~nm}$ with respect to the active zone by using a distance transform. . . . . . . . . . . . . . . . . . . 24

7 Estimated lambda of the parametric fit per synapse for: (a) 2D estimated distances , (b) 3D estimated distances. Solid lines indicate the median of parameter $\lambda$ for each group of rats, and the dashed indicates the median of the absolute difference of each point to the group median. . . . . . . . . . . . . . 25

8 Examples of active zones from the same slice and visualized at same resolution. (a) shows an apparently wide zone, while (b) shows a more narrow zone. . . . . . . . . . . . . . 26 
9 Comparison of synapses with different active zone shape: (a) a section from S7, and (b) a section form S2. . . . . . . . 27

10 A section of S6, which has a great number of synaptic vesicles. 28 


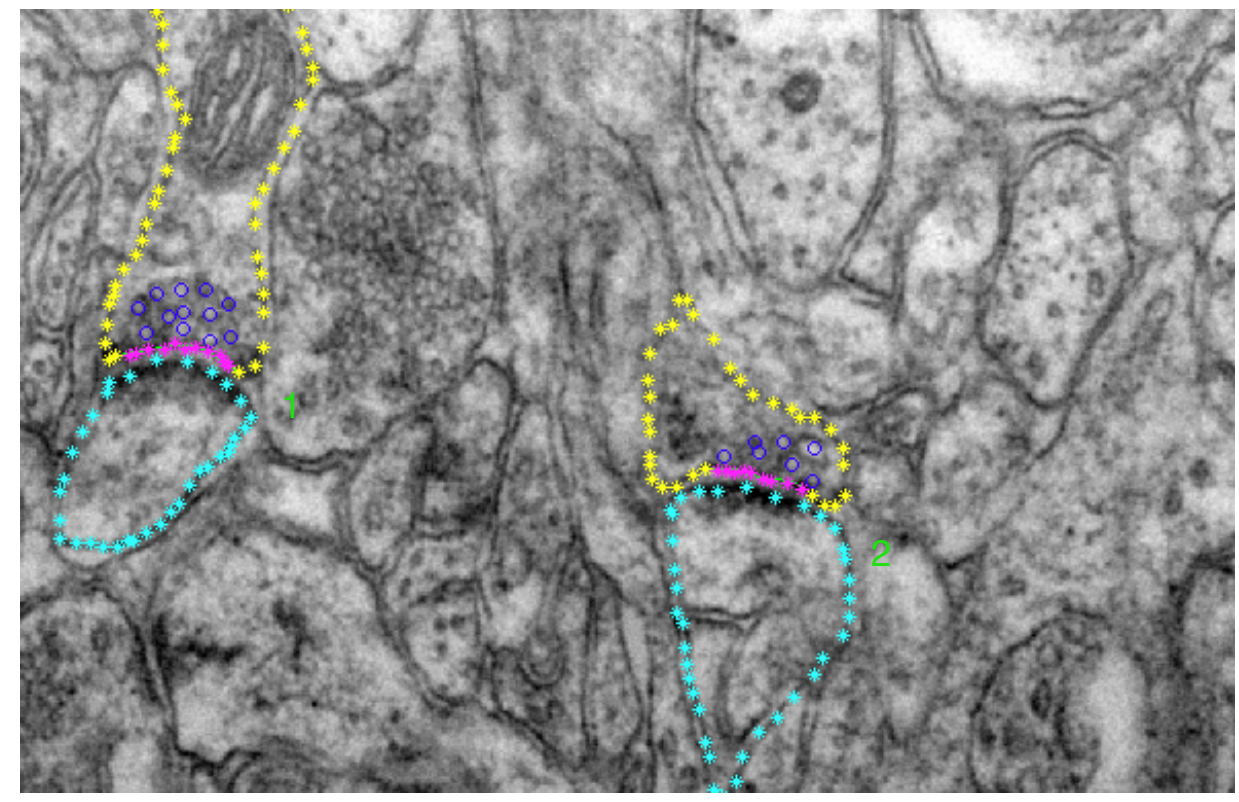

Figure 1: Example of section annotation. Typically, synapses present a presynaptic compartment (yellow annotation), separated from the post-synaptic terminal (cyan annotation) by an extracellular space termed synaptic cleft. Clustered within the presynaptic compartment are small translucent synaptic vesicles (blue annotation), where neurotransmitter is stored. Neurotransmitters are released upon arrival of action potential through fusion of synaptic vesicles to the active zone of the presynaptic membrane. 


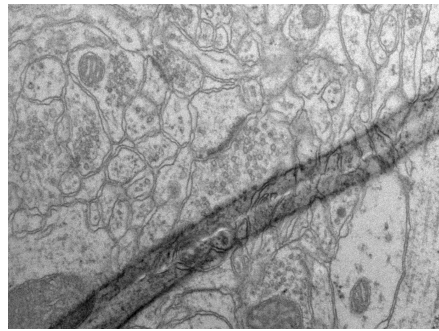

(a)

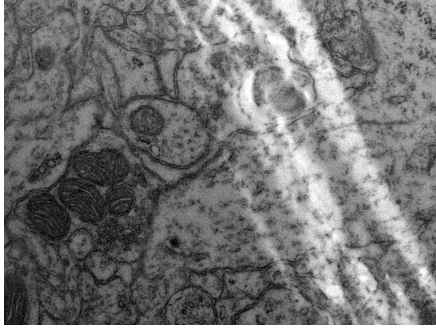

(b)

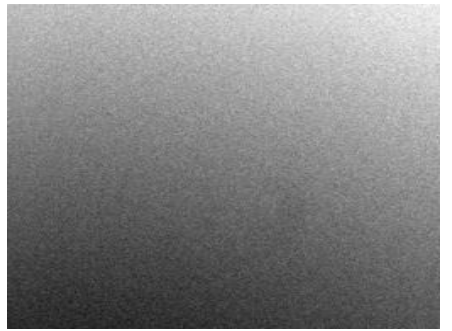

(c)

Figure 2: Examples of noise in the imaging system: from (a) the sectioning knife, (b) the formvar film, and (c) spatial inhomogeneity. 


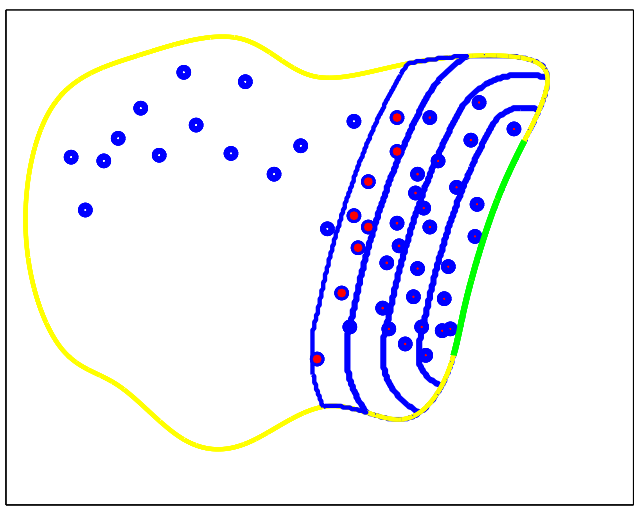

Figure 3: An example of producing closed parallel curves in a section. The active zone is shown in green, pre-synaptic terminal in yellow and vesicles are shown in red and blue respectively indicating location inside and outside of the closed parallel curve. 


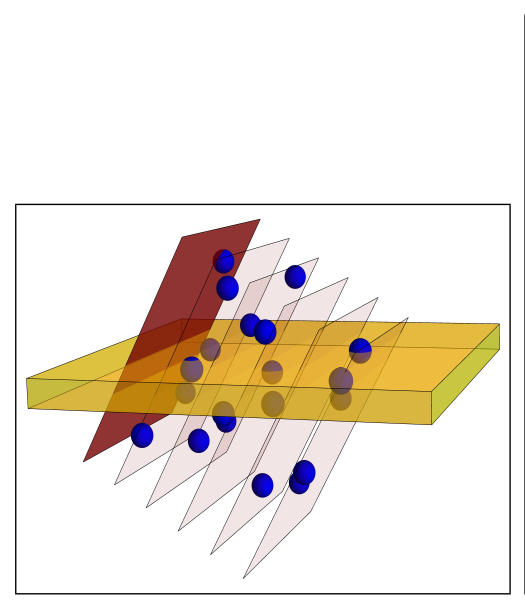

(a)

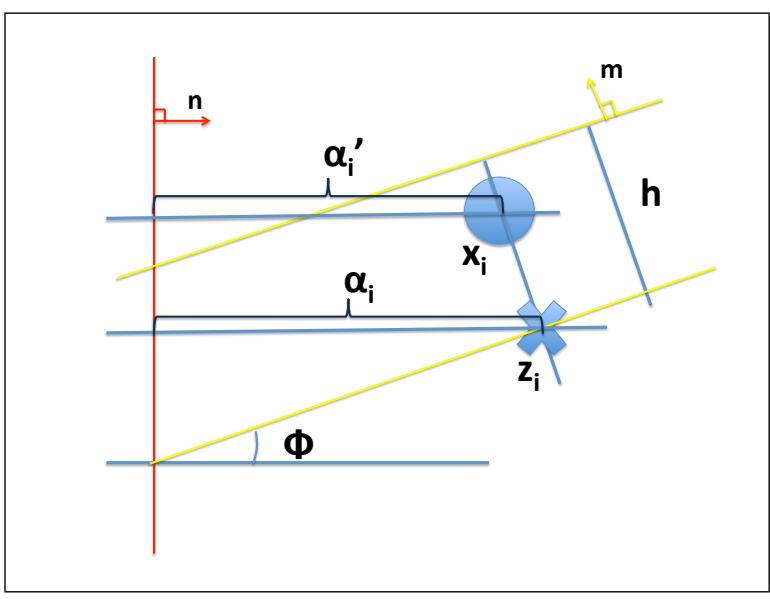

(b)

Figure 4: The figure shows a simplified synaptic region in 3D: a) A 3D visualisation of slicing and intersection, where the yellow thick horizontal plane illustrates a section, the active zone is illustrated as the red plane, the iso-distanced planes from the active zone are transparent red planes, and the synaptic vesicles are illustrated as blue spheres. b) A $2 \mathrm{D}$ visualisation of a similar situation but focussing on a single vesicle and as seen from the side. 


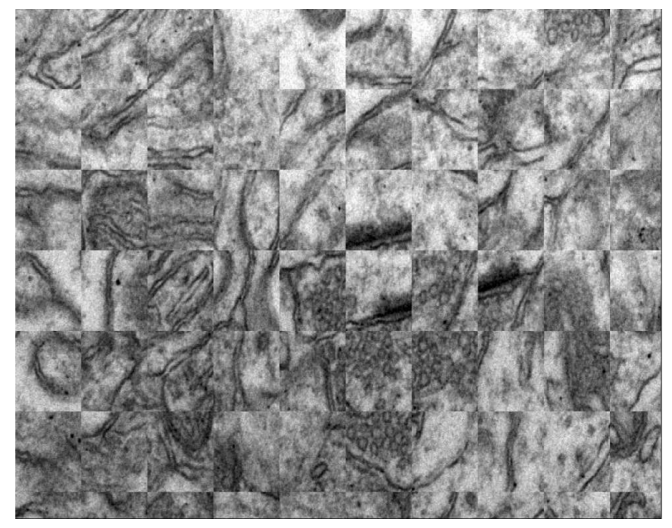

(a) Unregistered

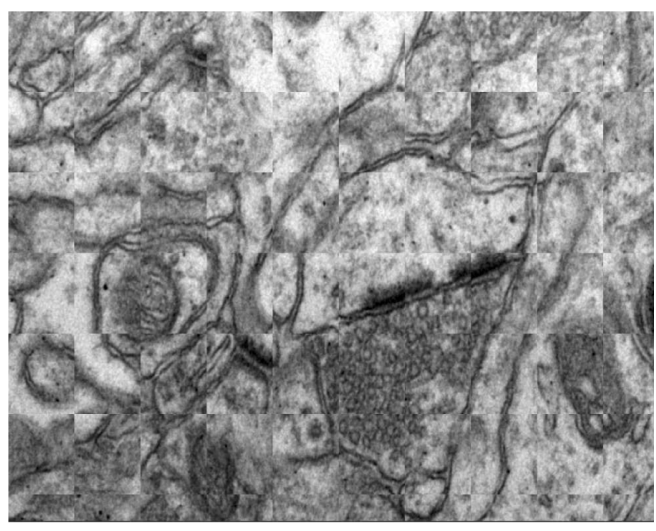

(b) Registered

Figure 5: Using chessboard visualisation to evaluate the registration results of two images. (a) The images before and (b) after registration. 


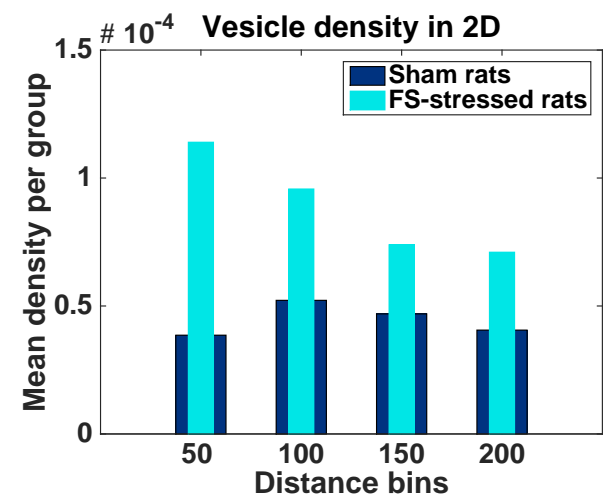

(a)

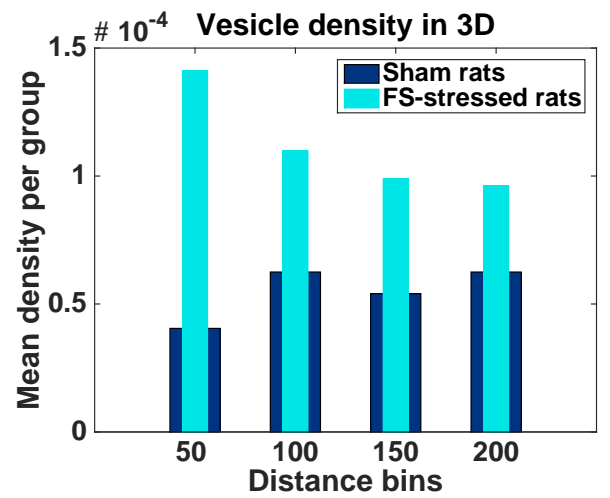

(b)

Figure 6: Histogram of synaptic vesicle density measured in (a) 2D and (b) 3D. The densities are measured in volume of interests with distance from $50 \mathrm{~nm}$ to $200 \mathrm{~nm}$ with respect to the active zone by using a distance transform. 


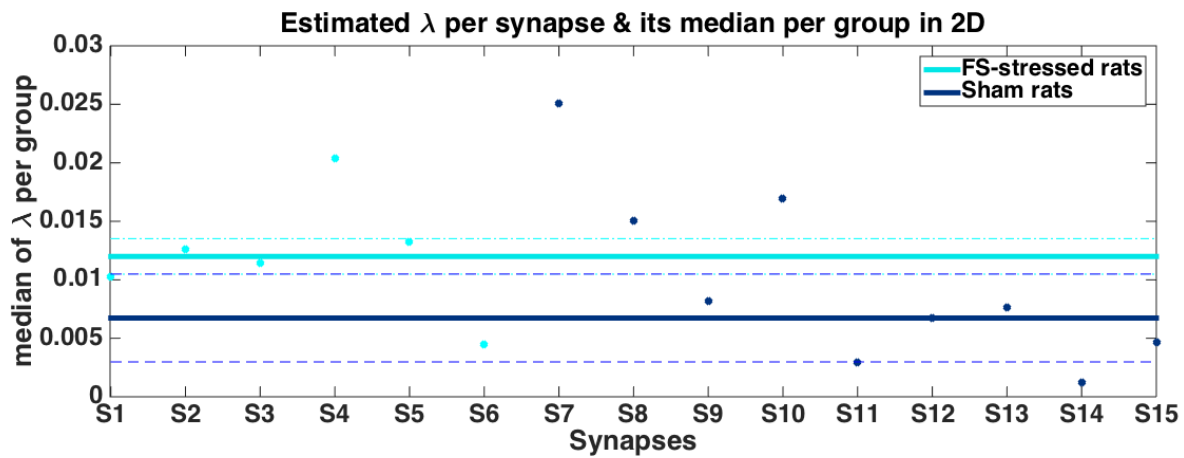

(a)

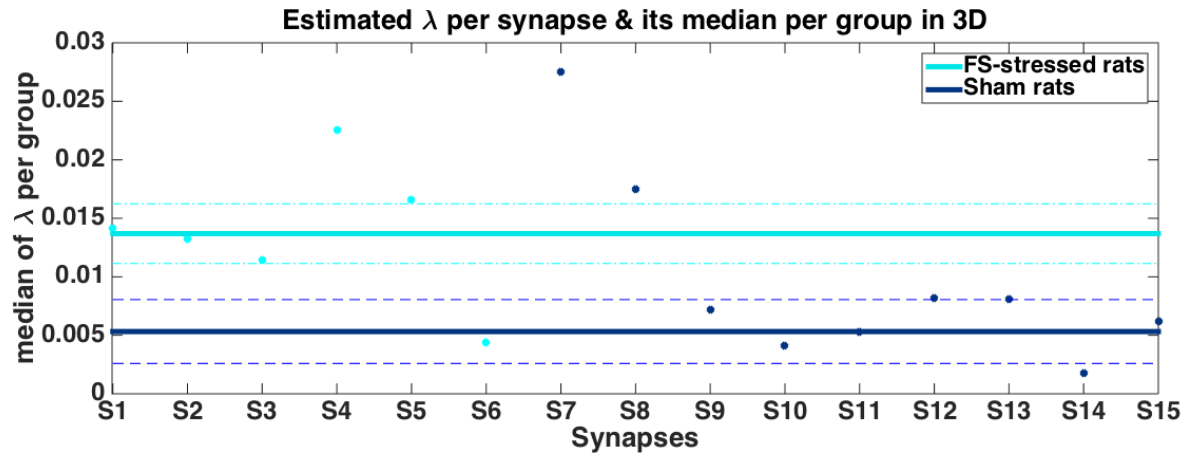

(b)

Figure 7: Estimated lambda of the parametric fit per synapse for: (a) $2 \mathrm{D}$ estimated distances , (b) 3D estimated distances. Solid lines indicate the median of parameter $\lambda$ for each group of rats, and the dashed indicates the median of the absolute difference of each point to the group median. 


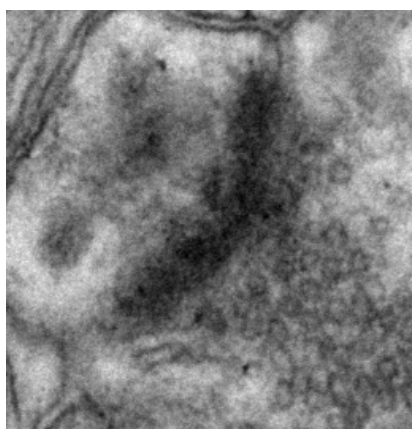

(a)

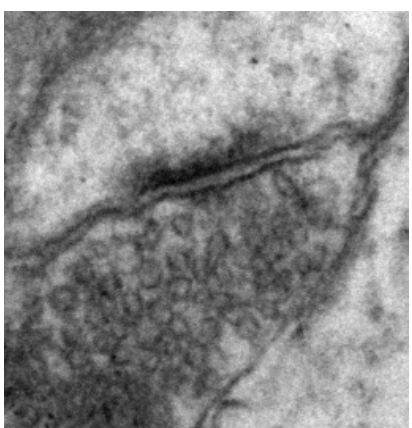

(b)

Figure 8: Examples of active zones from the same slice and visualized at same resolution. (a) shows an apparently wide zone, while (b) shows a more narrow zone. 


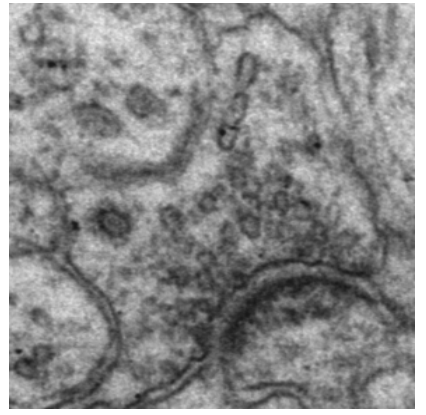

(a)

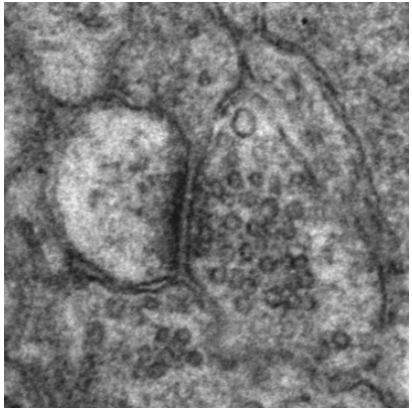

(b)

Figure 9: Comparison of synapses with different active zone shape: (a) a section from S7, and (b) a section form S2. 


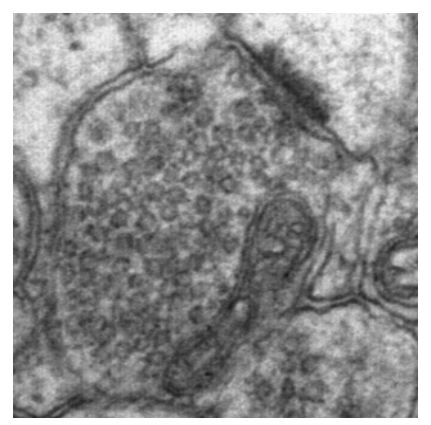

Figure 10: A section of S6, which has a great number of synaptic vesicles. 


\section{List of Tables}

1 Estimated average slope of the active zone with respect to the sectioning angle in sham and FS-stress rats. The angles are presented in degree unit. . . . . . . . . . . . . . . . . . . . 30

2 Simulations of randomly placed vesicles and their estimation in section and using the $3 \mathrm{D}$ reconstruction. The variable $n$ denotes the number of points in the section, $\bar{\lambda}_{2 \mathrm{D}}^{-}$the average estimated $\lambda$ when assuming only $2 \mathrm{D}$ information, and $\lambda_{3 \mathrm{D}}^{-}$ when utilising the 3D information from consecutive sections. . 31 


\begin{tabular}{|l|lllllllll|}
\hline$\theta$ (Sham) & 88.98 & 81.95 & 120.8 & 90.8 & 91.9 & 65.5 & 102.8 & 76.4 & 75 \\
\hline$\theta$ (FS-stress) & 123.15 & 85.75 & 96.71 & 71.9 & 76.29 & 93.78 & & & \\
\hline
\end{tabular}

Table 1: Estimated average slope of the active zone with respect to the sectioning angle in sham and FS-stress rats. The angles are presented in degree unit. 


\begin{tabular}{r|rrr}
$\phi$ & $n \pm \mathrm{std}$ & $\lambda_{2 \mathrm{D}}^{-} \pm \mathrm{std}$ & $\lambda_{3 \mathrm{D}}^{-} \pm \mathrm{std}$ \\
\hline 0.0 & $1056.2 \pm 32.2$ & $0.0098 \pm 0.0011$ & $0.0098 \pm 0.0011$ \\
10.0 & $994.3 \pm 30.3$ & $0.0097 \pm 0.0012$ & $0.0098 \pm 0.0012$ \\
20.0 & $940.0 \pm 31.5$ & $0.0093 \pm 0.0012$ & $0.0099 \pm 0.0013$ \\
30.0 & $896.6 \pm 31.5$ & $0.0087 \pm 0.0013$ & $0.0101 \pm 0.0015$ \\
40.0 & $847.6 \pm 27.0$ & $0.0078 \pm 0.0015$ & $0.0103 \pm 0.0019$ \\
50.0 & $790.3 \pm 24.8$ & $0.0067 \pm 0.0017$ & $0.0109 \pm 0.0028$ \\
60.0 & $681.0 \pm 26.3$ & $0.0047 \pm 0.0020$ & $0.0113 \pm 0.0048$
\end{tabular}

Table 2: Simulations of randomly placed vesicles and their estimation in section and using the $3 \mathrm{D}$ reconstruction. The variable $n$ denotes the number of points in the section, $\lambda_{2 \mathrm{D}}^{-}$ the average estimated $\lambda$ when assuming only $2 \mathrm{D}$ information, and $\lambda_{3 \mathrm{D}}^{-}$when utilising the $3 \mathrm{D}$ information from consecutive sections. 